\title{
NUMERICAL ANALYSES ON THE PREDICTION OF NUSSELT NUMBERS FOR UPWARD AND DOWNWARD FLOWS OF WATER IN A SMOOTH PIPE: EFFECTS OF BUOYANCY AND PROPERTY VARIATIONS
}

\author{
A.S. Dalkilic ${ }^{1, *}$, A. Çebi ${ }^{1}$, A. Celen ${ }^{1}$
}

\begin{abstract}
This paper includes the Artificial Neural Network (ANN) solution as one of the numerical analyses to investigate the buoyancy and property variation effects calculating Nusselt numbers during the upward and downward flow of water in a smooth pipe. Available data in the literature (Parlatan et al.) has been used in the analyses to show ANN's success ratio of predictability on the measured pipe length's averaged Nusselt numbers $\left(\mathrm{Nu}_{\text {avg }}\right)$ and forced convection's Nusselt numbers $\left(\mathrm{Nu}_{\mathrm{o}}\right)$. Mixed convective flow conditions were valid for Reynolds numbers ranging from 4000 to 9000 with Bond numbers smaller than 1.3. Dimensionless values of Reynolds number, Grashof number, Prandtl number, Bond number, Darcy friction factor, isothermal friction factor in forced convection, ratio of dynamic viscosities, and a Parlatan et al.'s friction factor were the inputs while $\mathrm{Nu}_{\text {avg }}$ and $\mathrm{Nu}_{\mathrm{o}}$ were the outputs of ANN analyses. All data was properly separated for test/training/validation processes. The ANNs performances were determined by way of relative error criteria with the practice of unknown test sets. As a result of analyses, outputs were predicted within the deviation of $\pm 5 \%$ accurately, new correlations were proposed using the inputs, and importance of inputs on the outputs were emphasized according to dependency analyses showing the importance of buoyancy influence $\left(\mathrm{Gr}_{\mathrm{T}}\right)$ and the effects of temperature-dependent viscosity variations under mixed convection conditions in aiding and opposing transition and turbulent flow of water in a test tube.
\end{abstract}

Keywords: Natural Convection, Single Phase Flow, Buoyancy and Property Variation, Friction Factor, Nusselt Number

\section{INTRODUCTION}

Mixed convection conditions occur when both forced and free convection have no negligible effects on flow. Mixed convection is nearly always the case in laminar pipe flows. If low external pressure gradient and low flow velocity, which are encountered in laminar flow, combine with the sufficient amount of density difference due to temperature change, mixed convection currents may form that modify the flow pattern significantly. Mixed convection has received remarkable interest over the years from various areas, mainly nuclear engineering and electronic cooling.

In vertical pipes, mixed convection is named according to the acting direction of the density-based pressure gradients. If flow is in upward direction and cools as it progresses, density-based pressure gradients are counter current to the flow direction and named as opposing flow. Opposite condition (aiding flow) is true when fluid is flowing upward and heated along the channel because density-based pressure gradients will be in upward direction this time. It also should be noted that aiding and opposing flow conditions will also occur in downward flow (downward cooling and downward heating for aiding and opposing flows respectively).

Tanaka et al. [1] found out the buoyancy and acceleration effects resulted in reduced shear stress near wall zone in turbulent forced convection in vertical tubes. These effects were found to be the reason behind the increment of friction; however, they have no remarkable influence over the heat transfer. In his experimental research with nitrogen in down flow condition, Easby [2] observed an increase in Stanton number due to buoyancy and shear stress distribution. His results also showed lower values as compared with previous correlations. Saylor and Joye [3] developed a method to compute the hydrostatic pressure difference that occurred due to temperature difference along the vertical pipe axis in mixed convection flows. Their improved method integrates the 
temperature along the pipe axis to have more reliable density and hydrostatic pressure values. Parlatan et al. [4] investigated friction factor and heat transfer characteristics of aiding and opposing flows in their experimental study. Friction factor was found to deviate from isothermal conditions by about $25 \%$ depending on the type of the flow. Depending on whether the flow is opposing or aiding type, alteration of friction factor deviates when viscosity variation is taken into consideration. They also found out that the heat transfer coefficient enhances in opposing flow, while it first decreases and then regains in aiding flow. You et al. [5] analyzed the vertical flow of heated air with direct numerical solution. They observed an increase and later a decrease in friction and Nusselt number with increasing heat flux while in upward flow conditions. In downward flow, no deviation is observed with friction, but with increasing heat flux an increase in Nusselt number is present, which leads to the conclusion that the velocity and temperature may have dissimilarity due to buoyancy.

Joye [6] experimentally investigated the laminar mixed flow in vertical pipe and derived an equation to predict the pressure drop as a function of flow rate. The equation can be used for Grashof number up to 108 and Reynolds number up to 11000. Busedra and Soliman [7] carried out a numerical study for mixed convection in inclined semicircular ducts for both aiding and opposing flow conditions under uniform heat flux and uniform wall temperature boundary assumptions. For upward flow conditions, friction factor was found to have an increasing characteristic under both boundary types but to have higher values in uniform heat flux conditions in high Grashof numbers. However, in downward flows, friction factor is always higher in uniform heat flux boundary conditions. Voicu et al. [8] numerically investigated the temperature and velocity profile of aqueous glycol solution in a vertical double pipe parallel heat exchanger under laminar mixed convection condition. They found out the Richardson number has influence over the velocity and temperature profile of the inner flow. Flow reversal in inner flow is observed above Richardson number 1; however, this situation diminishes as the flow progresses on because temperature difference along the radial direction decreases. Friction factor values were found to be related to Richardson number near the inlet region while they approach the forced convection regime values along the pipe length. Kang and Chung [9] made an experimental study to determine the influence of height-diameter ratio of a vertical tube over buoyancy effect. They recommended using the heated length as the characteristic length for the Grashof number because diameter was found to be inappropriate and cannot respond to the variety of the results due to length change. They used fixed diameter with varying pipe heights and fixed pipe height with varying diameters to identify the concept. Tam et al. [10] carried out an ANN analysis on laminar and turbulent heat transfer in horizontal tube to determine the importance of the different variables effecting heat transfer performance. Reynolds and Prandtl numbers are found to have the largest impact on the heat transfer for turbulent flow while Graetz and Rayleigh numbers are the most important parameters for laminar flow. Selimefendigil and Öztop [11] numerically investigated mixed flow characteristics of a square cavity with rotational cylinder in the center. The working fluid is water- $\mathrm{Al}_{2} \mathrm{O}_{3}$ nanoparticles mixture. GRNN method is the best procedure to determine the output, which was averaged Nusselt number. Their inputs are Reynolds number, Grashof number, volume fraction, and cylinder rotation speed. Generally all inputs are found to act as an enhancer to heat transfer if increased in certain amounts.

In this study, an artificial neural network analysis of Parlatan et al.'s [4] experimental data was carried out as one of rare studies of literature on this subject. New correlations were formed upon acquiring ANN data using dimensionless numbers of Reynolds number, Grashof number, Prandtl number, Bond number, Darcy friction factor, isothermal friction factor in forced convection, ratio of dynamic viscosities, and Parlatan et al.'s friction factor, and dependency analysis has been performed among inputs to determine the parameters' importance. Apart from other most in-tube forced convection studies, all analyses have the dimensionless numbers regarding buoyancy influence $\left(\mathrm{Gr}_{\square \mathrm{T}}\right)$ and the effects of temperature-dependent viscosity variations $\left(\mu_{\mathrm{w}} / \mu_{\mathrm{b}}\right)$ to benefit from one of the artificial intelligence techniques numerically.

\section{DATA REDUCTION}

Input and output parameters were selected from the dimensionless numbers used in Parlatan et al.'s study [4]. Inputs of the ANN model were Reynolds number, Prandtl number, Grashof number, Bond number, Darcy friction factor, isothermal friction factor in forced convection, ratio of dynamic viscosities, and Parlatan et al.'s friction factor, which was obtained from the experiments [4]. Inputs of the ANN model were given as follows:

Reynolds, Prandtl, Grashof $(\Delta \mathrm{T})$, Buoyancy, and Darcy friction factor numbers are expressed respectively: 


$$
\begin{aligned}
\operatorname{Re} & =\frac{\rho V D}{\mu} \\
\operatorname{Pr} & =\frac{v}{\alpha} \\
\mathrm{Gr}_{\Delta \mathrm{T}} & =\frac{\beta g\left(\mathrm{~T}_{\mathrm{w}}-\mathrm{T}_{\mathrm{b}}\right) \mathrm{D}^{3}}{v^{2}} \\
\mathrm{Bo} & =\frac{8 \times 10^{4} \mathrm{Gr}_{\mathrm{q}}}{\operatorname{Re}^{3.425} \operatorname{Pr}^{0.8}} \\
\mathrm{f} & =\frac{\Delta \mathrm{P} \cdot 2 \mathrm{D}}{\rho L \mathrm{~V}^{2}}
\end{aligned}
$$

However, $\mathrm{Gr}_{\Delta \mathrm{T}}$ was used in ANN model in accordance with Parlatan et al.'s experiments. Outputs of the ANN model were averaged with Nusselt numbers over pipe length and Nusselt number in forced conditions. Nusselt number is defined as:

$$
\mathrm{Nu}=\frac{\mathrm{q}^{\prime \prime} \mathrm{D}}{\mathrm{k}\left(\mathrm{T}_{\mathrm{w}}-\mathrm{T}_{\mathrm{b}}\right)}
$$

Since the ANNs are one of the generally used and established models to examine the formula between linear or non-linear input-output patterns, they try to generalize the training group and then estimate the test group. Performance of ANNs is determined with the achievement of the estimation. It should be noted that extensive knowledge of the use of ANN methods on the single- and two-phase flows, method of least squares, error analyses' calculation procedure including R square error, proportional error, and Mean square error can be seen from authors' previous publications [12-19].

\section{RESULTS AND DISCUSSION}

In dimensional examination, a dimensionless value is an amount without a related physical dimension. Dimensionless numbers are commonly used in many scientific areas. There are a lot of well-known quantities, such as Reynolds number, which is the ratio of inertial forces to viscous forces in a fluid. It is also defined as the ratio of total momentum transferred to the molecular transferred. The flow is smooth, continues, streamlines at low Re numbers. By contrast, the flow has eddies, vortices, and discontinues at high Re numbers. Prandtl number, which is the ratio between the momentum diffusivity to thermal diffusivity, depends on the state and type of the fluid and is independent from any length dimension. Grashof number, which is the ratio of the buoyancy to viscous force acting on a fluid, is a dominant one for natural convection. Bond number is the ratio of gravitational force to surface tension force. Nusselt number is the ratio of convective heat transfer across to boundary layer of the fluid to the conductive heat transfer. In this study, Nusselt numbers were determined numerically using the ANN analyses including various dimensionless numbers. The experimental data of Parlatan et al. [4] was shown in Tables 1 and 2 for opposing and aiding turbulent mixed convection flow conditions respectively. The range of experiments was $4160 \leq \operatorname{Re} \leq 9060,0.30 \leq \mathrm{Gr}_{\Delta \mathrm{T}} \times 10^{-6} \leq 6.93,4.28 \leq \operatorname{Pr} \leq 5.43$, and $0.03 \leq \mathrm{Bo} \leq 1.27$ for water in a vertical pipe. 
Table 1. Inputs and outputs of numerical analyses having the experimental database of upward flow from Parlatan et al. [4]'s study.

\begin{tabular}{|l|l|l|l|l|l|l|l|l|l|l|}
\hline Exp & $\begin{array}{c}\text { Input 1 } \\
\mathrm{Re}\end{array}$ & $\begin{array}{c}\text { Input 2 } \\
\mathrm{Gr}_{\Delta \mathrm{T} \times 10^{-}} \\
6\end{array}$ & $\begin{array}{c}\text { Input 3 } \\
\mathrm{Pr}\end{array}$ & $\begin{array}{c}\text { Input 4 } \\
\text { Bo }\end{array}$ & $\begin{array}{c}\text { Input 5 } \\
\mathrm{f}\end{array}$ & $\begin{array}{c}\text { Input 6 } \\
\mathrm{f}_{\mathrm{o}}\end{array}$ & $\begin{array}{c}\text { Input 7 } \\
\mu_{\mathrm{w}} / \mu_{\mathrm{b}}\end{array}$ & $\begin{array}{c}\text { Input 8 } \\
\mathrm{f}_{\mathrm{vp}}\end{array}$ & $\begin{array}{c}\text { Output 1 } \\
\mathrm{Nu}_{\text {avg }}\end{array}$ & $\begin{array}{c}\text { Output } \\
2 \\
\mathrm{Nu}_{\mathrm{o}}\end{array}$ \\
\hline $1 \mathrm{U}$ & 7513 & 4.73 & 4.97 & 0.138 & 0.0287 & 0.0328 & 0.51 & 0.0275 & 24.3 & 49.390 \\
\hline $2 \mathrm{U}$ & 7391 & 2.11 & 5.06 & 0.114 & 0.0306 & 0.0330 & 0.7 & 0.0307 & 41.6 & 49.056 \\
\hline $3 \mathrm{U}$ & 7200 & 0.988 & 5.22 & 0.061 & 0.0317 & 0.0333 & 0.84 & 0.0323 & 46.8 & 48.397 \\
\hline $4 \mathrm{U}$ & 7031 & 0.474 & 5.36 & 0.031 & 0.0328 & 0.0336 & 0.91 & 0.0329 & 47.1 & 47.865 \\
\hline $5 \mathrm{U}$ & 6069 & 0.538 & 5.38 & 0.05 & 0.0344 & 0.0355 & 0.9 & 0.0348 & 39.7 & 42.688 \\
\hline $6 \mathrm{U}$ & 5012 & 0.653 & 5.4 & 0.094 & 0.0409 & 0.0378 & 0.87 & 0.0371 & 31.5 & 36.713 \\
\hline $7 \mathrm{U}$ & 4163 & 1.08 & 5.36 & 0.151 & - & - & - & - & 16.6 & 31.499 \\
\hline $8 \mathrm{U}$ & 4143 & 0.405 & 5.38 & 0.089 & 0.0408 & 0.0402 & 0.92 & 0.0394 & 24.8 & 31 \\
\hline $9 \mathrm{U}$ & 4990 & 0.304 & 5.43 & 0.047 & 0.0388 & 0.0380 & 0.94 & 0.0376 & 33.8 & 36.540 \\
\hline $10 \mathrm{U}$ & 5087 & 1.61 & 5.31 & 0.139 & 0.0328 & 0.0377 & 0.75 & 0.0354 & 20.1 & 36.948 \\
\hline $11 \mathrm{U}$ & 4210 & 2.06 & 5.29 & 0.249 & - & - & - & - & 14.3 & 31.567 \\
\hline $12 \mathrm{U}$ & 6218 & 1.05 & 5.24 & 0.088 & 0.035 & 0.0352 & 0.82 & 0.0338 & 37.6 & 43.168 \\
\hline $13 \mathrm{U}$ & 5268 & 3.13 & 5.1 & 0.227 & - & - & - & - & 17.8 & 37.552 \\
\hline $14 \mathrm{U}$ & 4391 & 3.24 & 5.04 & 0.497 & - & - & - & - & 19.3 & 35.873 \\
\hline $15 \mathrm{U}$ & 4578 & 4.62 & 4.82 & 0.771 & - & - & - & - & 28.1 & 33.733 \\
\hline $16 \mathrm{U}$ & 5462 & 4.48 & 4.9 & 0.398 & 0.0394 & 0.0368 & 0.47 & 0.0302 & 22.7 & 38.151 \\
\hline $17 \mathrm{U}$ & 6341 & 2.82 & 5.12 & 0.14 & 0.0312 & 0.0349 & 0.65 & 0.0317 & 24 & 43.557 \\
\hline $18 \mathrm{U}$ & 6689 & 5.54 & 4.81 & 0.27 & 0.0436 & 0.0343 & 0.46 & 0.0278 & 24.8 & 44.604 \\
\hline $19 \mathrm{U}$ & 4658 & 2.59 & 5.19 & 0.259 & - & - & - & - & 16.3 & 34.100 \\
\hline $20 \mathrm{U}$ & 4837 & 3.54 & 4.97 & 0.441 & 0.0471 & 0.0384 & 0.54 & 0.0330 & 21 & 34.710 \\
\hline $21 \mathrm{U}$ & 5220 & 6.93 & 4.55 & 0.92 & - & - & - & - & 27.8 & 36.010 \\
\hline
\end{tabular}

ANN analyses have 8 inputs mainly as dimensionless values of Reynolds number, Grashof number, Prandtl number, Bond number, Darcy friction factor, isothermal friction factor in forced convection, ratio of dynamic viscosities, and Parlatan et al.'s friction factor, while $\mathrm{Nu}_{\text {avg }}$ and $\mathrm{Nu}_{\mathrm{o}}$ were the outputs of $\mathrm{ANN}$ analyses. Fig. 1 shows ANN results for the pipe length averaged and forced convection's Nusselt numbers within the deviation band of $\pm 20 \%$ and $\pm 10 \%$ respectively. Table 3 shows the error rates of ANN analyses for the predicted outputs. According to this table, it is possible to derive many results. The effect of input numbers after first 4 inputs on the outputs were found to have almost negligible effect. For instance, the first 4 inputs have prediction capacity of output one as 0.965 whereas all inputs have increased these values to 0.973 in this table. This result shows the importance of first 4 inputs in the analyses. All inputs of analyses were found to have significant importance with their individual error values in this table. Generally, Table 3 shows the possibility of the prediction by ANN analyses on the investigated subject using proper inputs clearly.

Tables 4 and 5 include all proposed correlations by means of the method of least squares for the prediction of outputs 1 and 2 respectively. Number of inputs in these correlations decreases with increasing their labels. Correlation 1 has all inputs and correlation 6 has 3 inputs. As their success can be seen from Figs. 2 to 5, correlation 1 is the most predictive one due to its large number of inputs as expected. Figs. 2 and 3 illustrate the results of proposed correlations of 1, 2, 5, and 6 for output 1 while Figs. 4 and 5 depict the results of proposed correlations of $1,2,5$, and 6 for the output 2 , which was predicted more accurately than another one within the deviation band of $\pm 5 \%$. Table 6 shows the results of correlations 3 and 4 numerically. Accuracy of the proposed correlations increase with increasing input numbers as shown in related tables and figures. 
Journal of Thermal Engineering, Research Article, Vol. 5, No. 3, pp. 166-180, April, 2019

Table 2. Inputs and outputs of numerical analyses (Downward flow data from Parlatan et al. [4])

\begin{tabular}{|c|c|c|c|c|c|c|c|c|c|c|}
\hline Exp & $\begin{array}{c}\text { Input } 1 \\
\text { Re }\end{array}$ & $\begin{array}{c}\text { Input } 2 \\
\mathrm{Gr}_{\Delta \mathrm{T}} \mathrm{X} 10^{-6}\end{array}$ & $\begin{array}{c}\text { Input } 3 \\
\text { Pr }\end{array}$ & $\begin{array}{c}\text { Input } 4 \\
\text { Bo }\end{array}$ & $\begin{array}{c}\text { Input } 5 \\
\mathrm{f}\end{array}$ & $\begin{array}{c}\text { Input } 6 \\
f_{o}\end{array}$ & $\begin{array}{l}\text { Input } 7 \\
\mu_{\mathrm{w}} / \mu_{\mathrm{b}}\end{array}$ & $\begin{array}{c}\text { Input } 8 \\
\mathrm{f}_{\mathrm{vp}}\end{array}$ & $\begin{array}{c}\text { Output } 1 \\
\mathrm{Nu}_{\mathrm{avg}}\end{array}$ & $\begin{array}{c}\text { Output } 2 \\
\mathrm{Nu}_{\mathrm{o}}\end{array}$ \\
\hline $22 \mathrm{D}$ & 5154 & 1.09 & 5.23 & 0.181 & 0.0325 & 0.0401 & 0.82 & 0.0385 & 40.2 & 37.050 \\
\hline $23 \mathrm{D}$ & 5307 & 1.72 & 5.06 & 0.279 & 0.0309 & 0.0398 & 0.76 & 0.0378 & 42 & 37.701 \\
\hline $24 \mathrm{D}$ & 5646 & 3.14 & 4.74 & 0.498 & - & - & - & - & 48 & 38.709 \\
\hline $25 \mathrm{D}$ & 5956 & 4.95 & 4.43 & 0.73 & - & - & - & - & 52.1 & 39.710 \\
\hline $26 \mathrm{D}$ & 5112 & 0.74 & 5.28 & 0.119 & 0.34 & 0.4023 & 0.87 & 0.3943 & 38 & 37.037 \\
\hline $27 \mathrm{D}$ & 4653 & 0.815 & 5.19 & 0.174 & 0.0328 & 0.0413 & 0.87 & 0.0400 & 36 & 34.058 \\
\hline $28 \mathrm{D}$ & 4366 & 0.812 & 5.29 & 0.204 & - & - & - & - & 34.5 & 32.608 \\
\hline $29 \mathrm{D}$ & 4339 & 0.433 & 5.33 & 0.106 & 0.0367 & 0.0421 & 0.92 & 0.0412 & 32.3 & 32.527 \\
\hline $30 \mathrm{D}$ & 4671 & 0.424 & 5.31 & 0.083 & 0.0366 & 0.0413 & 0.92 & 0.0408 & 34.2 & 34.475 \\
\hline $31 \mathrm{D}$ & 4873 & 1.26 & 5.16 & 0.26 & 0.0321 & 0.0409 & 0.81 & 0.0393 & 38.1 & 34.890 \\
\hline $32 \mathrm{D}$ & 4527 & 1.35 & 5.08 & 0.334 & 0.0321 & 0.0416 & 0.8 & 0.0399 & 37.4 & 33.156 \\
\hline $33 \mathrm{D}$ & 4999 & 1.96 & 4.91 & 0.39 & - & - & - & - & 41.4 & 35.506 \\
\hline $34 \mathrm{D}$ & 6333 & 1.53 & 5.13 & 0.15 & 0.0299 & 0.0378 & 0.78 & 0.0359 & 47.1 & 43.530 \\
\hline $35 \mathrm{D}$ & 7357 & 1.37 & 5.18 & 0.088 & 0.0297 & 0.0360 & 0.79 & 0.0346 & 52.5 & 49.203 \\
\hline $36 \mathrm{D}$ & 8468 & 1.25 & 5.15 & 0.055 & 0.0289 & 0.0344 & 0.81 & 0.0330 & 58.3 & 55 \\
\hline $37 \mathrm{D}$ & 8640 & 1.9 & 5.03 & 0.082 & 0.0276 & 0.0341 & 0.75 & 0.0321 & 59.8 & 55.421 \\
\hline $38 \mathrm{D}$ & 7582 & 2.14 & 5 & 0.135 & 0.0275 & 0.0356 & 0.73 & 0.0335 & 54.8 & 49.863 \\
\hline $39 \mathrm{D}$ & 6606 & 2.47 & 4.89 & 0.234 & - & - & - & - & 50.7 & 44.395 \\
\hline $40 \mathrm{D}$ & 5048 & 3.01 & 4.73 & 0.637 & - & - & - & - & 44.2 & 35.473 \\
\hline $41 \mathrm{D}$ & 4868 & 3.89 & 4.67 & 0.756 & - & - & - & - & 43.8 & 34.272 \\
\hline $42 \mathrm{D}$ & 7702 & 2.78 & 4.91 & 0.173 & - & - & - & - & 56.6 & 50.311 \\
\hline $43 \mathrm{D}$ & 6710 & 3.19 & 4.8 & 0.302 & - & - & - & - & 52.8 & 44.707 \\
\hline $44 \mathrm{D}$ & 5293 & 4.97 & 4.6 & 0.782 & - & - & - & - & 47.2 & 36.140 \\
\hline $45 \mathrm{D}$ & 5020 & 4.14 & 4.51 & 0.983 & - & - & - & - & 46.9 & 34.766 \\
\hline $46 \mathrm{D}$ & 8740 & 2.5 & 4.97 & 0.108 & - & - & - & - & 61.4 & 55.818 \\
\hline $47 \mathrm{D}$ & 6993 & 4.34 & 4.57 & 0.39 & - & - & - & - & 55.8 & 45.551 \\
\hline $48 \mathrm{D}$ & 7930 & 3.73 & 4.75 & 0.223 & - & - & - & - & 58.7 & 50.910 \\
\hline 49D & 9059 & 3.41 & 4.77 & 0.139 & - & - & - & - & 63.7 & 56.723 \\
\hline $50 \mathrm{D}$ & 5518 & 5.15 & 4.38 & 0.987 & - & - & - & & 50.8 & 37.216 \\
\hline $51 \mathrm{D}$ & 5251 & 5.6 & 4.28 & 1.273 & - & - & - & 0.0275 & 50.4 & 35.492 \\
\hline
\end{tabular}

Table 3. Error rates of ANN analyses for the predicted outputs

\begin{tabular}{|c|c|c|c|c|c|c|c|c|}
\hline \multicolumn{9}{|c|}{ Output $1\left(\mathrm{Nu}_{\text {avg,exp }}\right)$} \\
\hline & $\begin{array}{c}\text { Input } \\
1 \\
(\mathrm{Re})\end{array}$ & $\begin{array}{c}\text { Input } 2 \\
\left(\mathrm{Gr}_{\Delta \mathrm{T}}\right. \\
\left..10^{-6}\right)\end{array}$ & $\begin{array}{c}\text { Input } \\
3 \\
(\mathrm{Pr})\end{array}$ & $\begin{array}{c}\text { Input } \\
4 \\
\text { (Bo) }\end{array}$ & $\begin{array}{c}\text { Input } \\
5 \\
\text { (f) }\end{array}$ & $\begin{array}{c}\text { Input } \\
6 \\
\left(f_{o}\right)\end{array}$ & $\begin{array}{l}\text { ANN inc. all } \\
\text { inputs }\end{array}$ & $\begin{array}{l}\text { ANN inc. inputs } \\
\text { from } 1 \text { to } 4\end{array}$ \\
\hline $\begin{array}{l}\text { Root Mean } \\
\text { Squared Error }\end{array}$ & 2.271 & 2.281 & 2.284 & 2.34 & 2.404 & 3.593 & 2.22 & 2.549 \\
\hline R-Squared Error & 0.958 & 0.957 & 0.957 & 0.955 & 0.953 & 0.928 & 0.973 & 0.965 \\
\hline \multicolumn{9}{|c|}{ Output $2\left(\mathrm{Nu}_{\mathrm{o}, \exp }\right)$} \\
\hline & $\begin{array}{c}\text { Input } \\
1 \\
(\mathrm{Re})\end{array}$ & $\begin{array}{c}\text { Input } 2 \\
\left(\mathrm{Gr}_{\Delta \mathrm{T}}\right. \\
\left..10^{-6}\right)\end{array}$ & $\begin{array}{c}\text { Input } \\
3 \\
(\mathrm{Pr})\end{array}$ & $\begin{array}{c}\text { Input } \\
4 \\
\text { (Bo) }\end{array}$ & $\begin{array}{c}\text { Input } \\
5 \\
\text { (f) }\end{array}$ & $\begin{array}{c}\text { Input } \\
6 \\
\left(f_{o}\right)\end{array}$ & $\begin{array}{l}\text { ANN inc. all } \\
\text { inputs }\end{array}$ & $\begin{array}{l}\text { ANN inc. inputs } \\
\text { from } 1 \text { to } 4\end{array}$ \\
\hline $\begin{array}{l}\text { Root Mean } \\
\text { Squared Error }\end{array}$ & 0.274 & 0.276 & 0.3 & 0.301 & 0.874 & 0.907 & 0.472 & 0.528 \\
\hline R-Squared Error & 0.998 & 0.998 & 0.998 & 0.998 & 0.985 & 0.984 & 0.996 & 0.994 \\
\hline
\end{tabular}



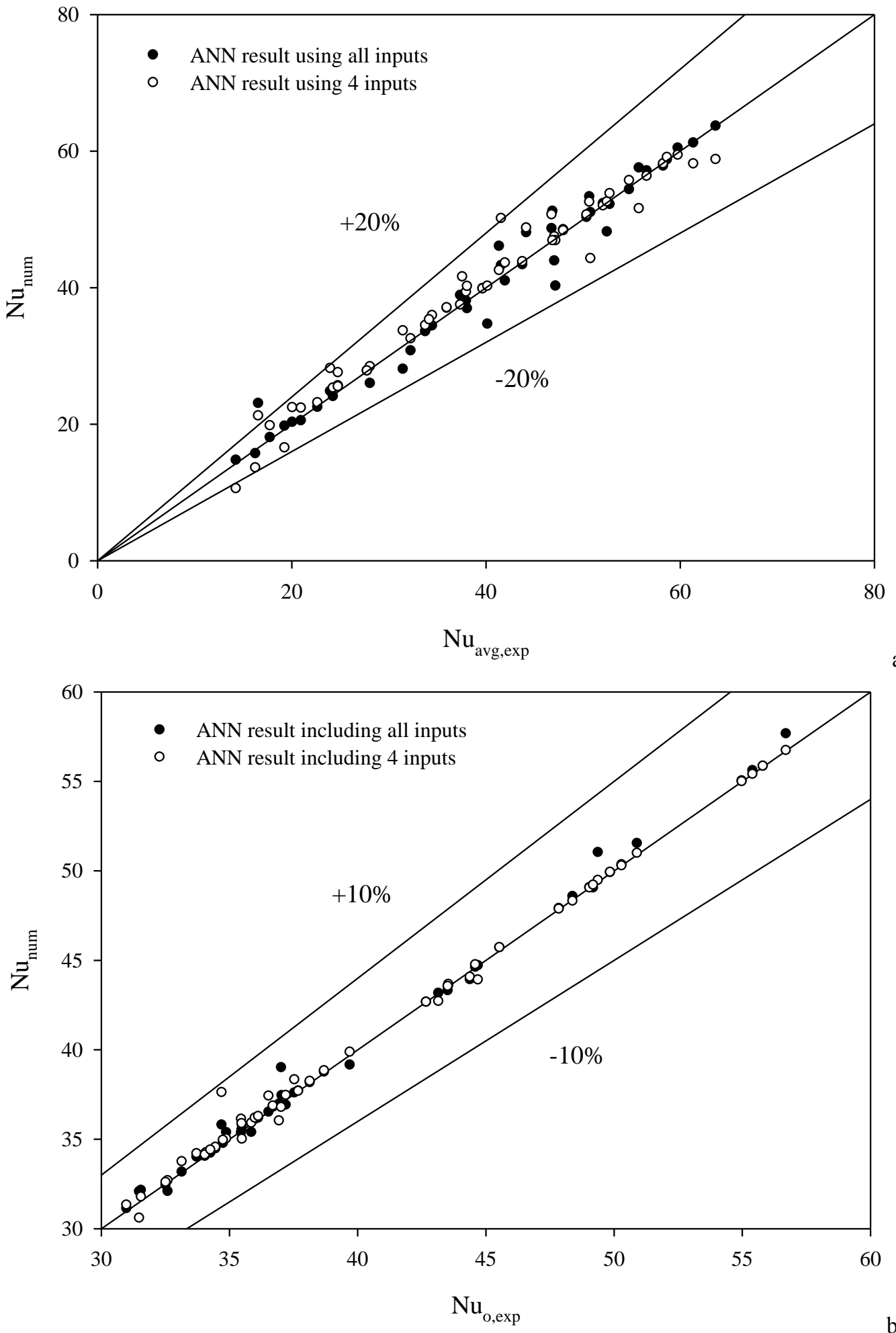

a)

Figure 1. ANN results for the pipe length averaged (a) and forced convection's (b) Nusselt numbers 


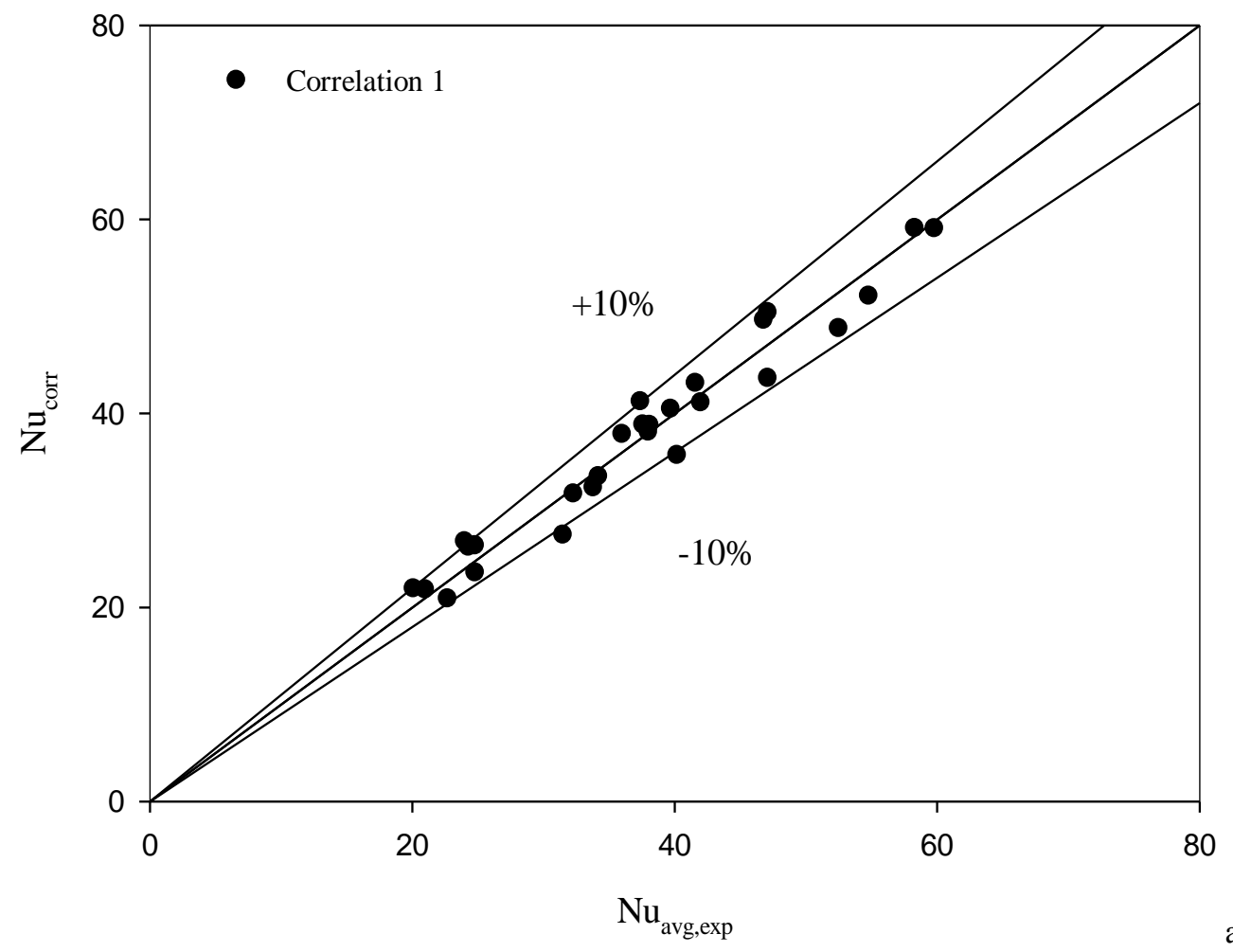

a)

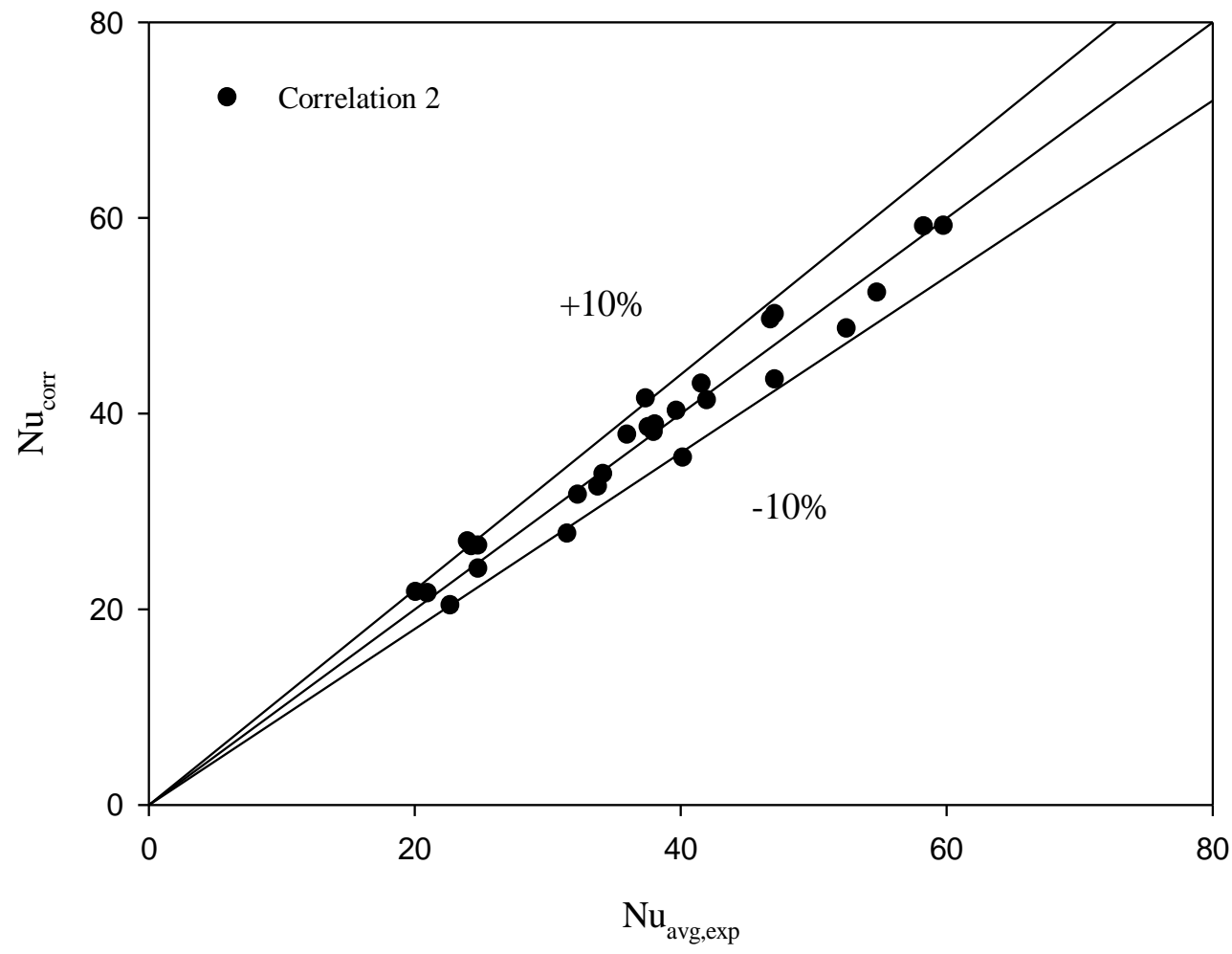

b)

Figure 2. Comparison of proposed correlations with output 1 experimental data 

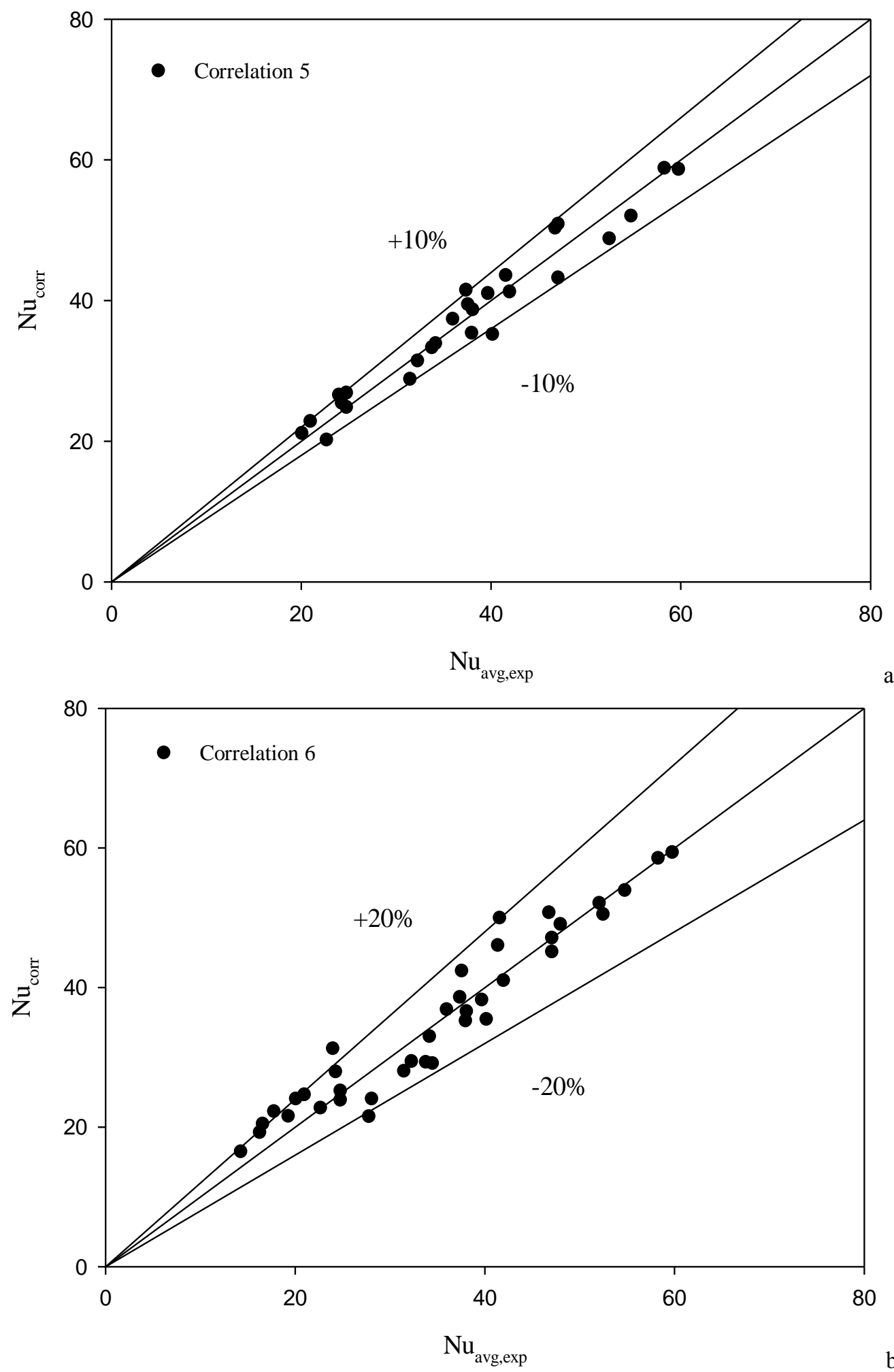

a)

Figure 3. Comparison of proposed correlations with output 1 experimental data 

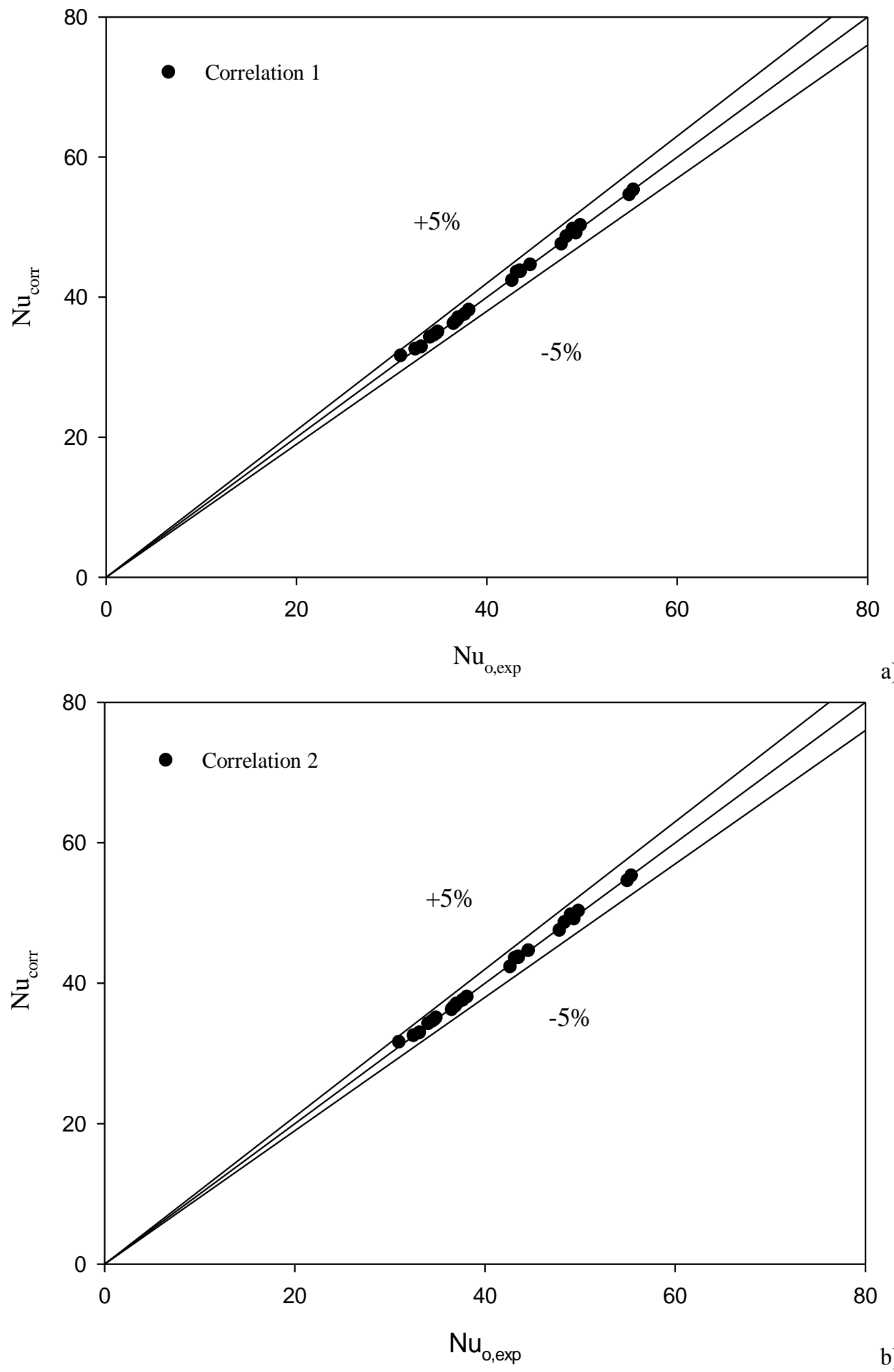

)

Figure 4. Comparison of proposed correlations with output 2 experimental data 

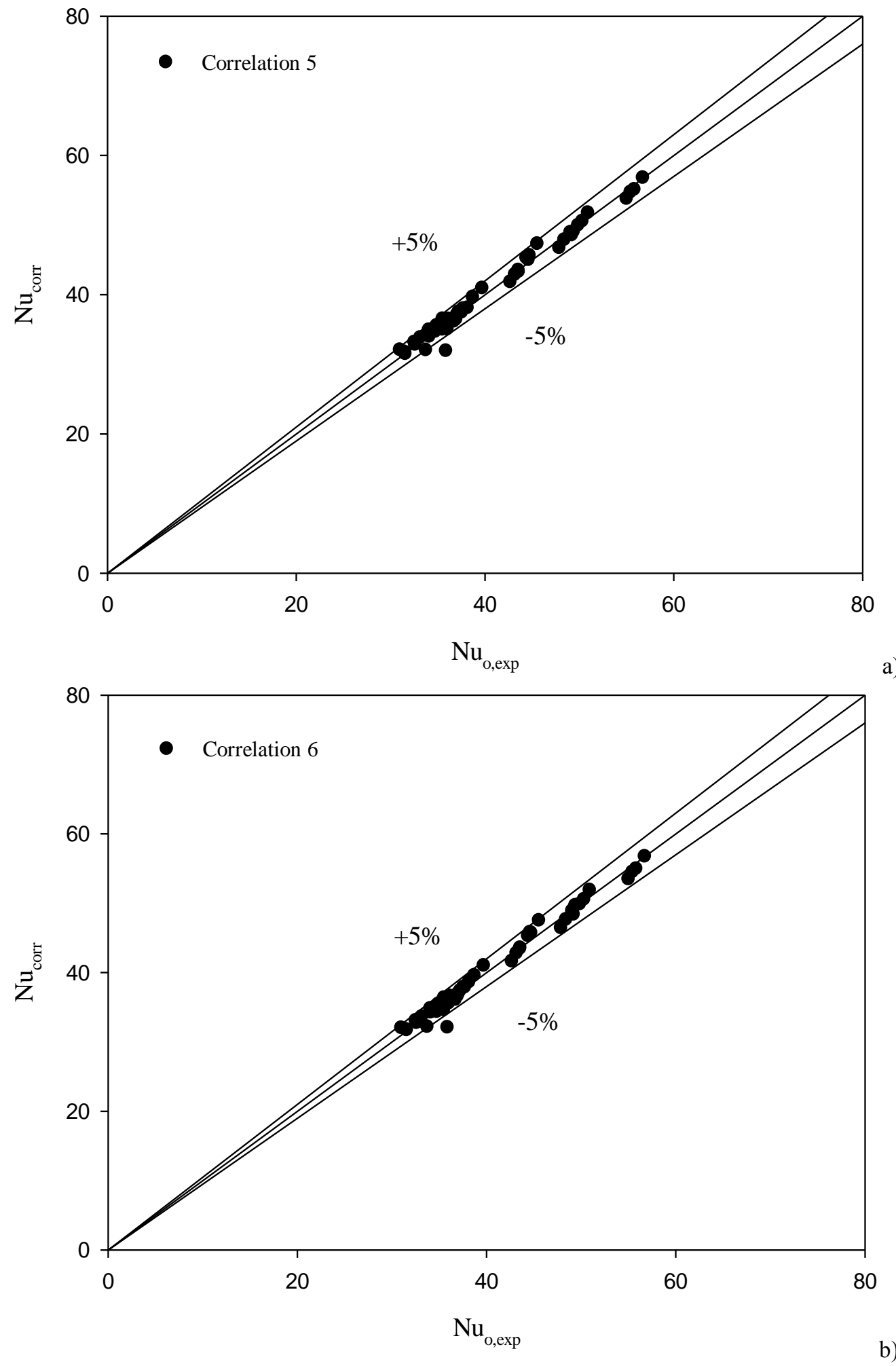

Figure 5. Comparison of proposed correlations with output 2 experimental data 
Table 4. Proposed correlations, their coefficients and p-values for output 1

\begin{tabular}{|c|c|c|c|c|c|c|c|c|c|}
\hline \multicolumn{10}{|c|}{ Correlation 1: b1/(1+ exp(b2*input $1+\mathrm{b} 3 *$ input $2+\mathrm{b} 4 *$ input $3+\mathrm{b} 5 *$ input $4+\mathrm{b} 6 *$ input5 $+\mathrm{b} 7 *$ input6 $+\mathrm{b} 8 *$ input $7+\mathrm{b} 9 *$ input8) $)$} \\
\hline & b1 & b2 & b3 & b4 & b5 & b6 & b7 & b8 & b9 \\
\hline Coefficient & 76.6042 & -0.0005 & 0.0683 & 1.6986 & -3.2181 & 9.8277 & -85.3757 & -6.6665 & 78.2192 \\
\hline p-value & $1.22 \mathrm{E}-05$ & 0.0005 & 0.6859 & 0.0007 & 0.0012 & 0.312 & 0.6205 & 0.0071 & 0.6478 \\
\hline \multicolumn{10}{|c|}{ Correlation $2: \mathrm{b} 1 /(1+\exp (\mathrm{b} 2 *$ input $1+\mathrm{b} 3 *$ input3 $+\mathrm{b} 4 *$ input $4+\mathrm{b} 5 *$ input5 + b6*input6 + b7*input7 + b8*input8) $)$} \\
\hline & b1 & b2 & b3 & b4 & b5 & b6 & b7 & $\mathrm{b} 8$ & b9 \\
\hline Coefficient & 78.2551 & -0.0005 & 1.6871 & -3.1923 & 8.375 & -33.5803 & -6.6123 & 26.6497 & - \\
\hline \multicolumn{10}{|c|}{ Correlation 3: b1/(1+ exp $(\mathrm{b} 2 *$ input $1+\mathrm{b} 3 *$ input3 $+\mathrm{b} 4 *$ input $4+\mathrm{b} 5 *$ input5 $+\mathrm{b} 6 *$ input6 $+\mathrm{b} 7 *$ input7) $)$} \\
\hline & b1 & $\mathrm{b} 2$ & b3 & b4 & b5 & b6 & b7 & b8 & b9 \\
\hline Coefficient & 79.1742 & -0.0004 & 1.6126 & -3.1975 & 7.6753 & -6.8591 & -6.181 & - & - \\
\hline p-value & $1.05 \mathrm{E}-05$ & 0.0002 & $6.11 \mathrm{E}-06$ & 0.0008 & 0.313 & 0.2837 & $4.35 \mathrm{E}-05$ & - & - \\
\hline \multicolumn{10}{|c|}{ Correlation $4: \mathrm{b} 1 /(1+\exp (\mathrm{b} 2 *$ input $1+\mathrm{b} 3 *$ input $3+\mathrm{b} 4 *$ input $4+\mathrm{b} 5 *$ input $6+\mathrm{b} 6 *$ input 7$))$} \\
\hline \multicolumn{10}{|c|}{ Correlation 5: b1/(1+ exp(b2*input1 + b3*input3 + b4*input4 + b5*input7)) } \\
\hline & b1 & b2 & b3 & b4 & b5 & b6 & b7 & b8 & b9 \\
\hline Coefficient & 74.4857 & -0.0005 & 1.7732 & -3.6349 & -6.9243 & - & - & - & - \\
\hline p-value & $3.50 \mathrm{E}-07$ & $5.27 \mathrm{E}-05$ & 8.32E-07 & 0.0001 & $4.61 \mathrm{E}-06$ & - & - & - & - \\
\hline Coefficient & 70.7088 & -0.0004 & 0.6426 & 3.3675 & -0.8131 & -15.8385 & - & - & - \\
\hline p-value & $1.81 \mathrm{E}-12$ & $3.67 \mathrm{E}-05$ & $1.34 \mathrm{E}-08$ & $1.65 \mathrm{E}-06$ & 0.0916 & $2.14 \mathrm{E}-05$ & - & - & - \\
\hline
\end{tabular}

Table 5. Proposed correlations, their coefficients and p-values for output 2

\begin{tabular}{|c|c|c|c|c|c|c|c|c|c|}
\hline \multicolumn{10}{|c|}{ Correlation 1: b1/(1+ exp(b2*input1 + b3*input2 + b4*input3 + b5*input4 + b6*input5 + b7*input6 + b8*input7 + b9*input8) } \\
\hline & b1 & $\mathrm{b} 2$ & b3 & $\mathrm{b} 4$ & b5 & b6 & b7 & $\mathrm{b} 8$ & b9 \\
\hline Coefficient & 73.4234 & -0.0003 & 0.079 & 0.1861 & 0.3411 & -1.3435 & -9.6328 & 0.5148 & 10.9657 \\
\hline p-value & $9.79 \mathrm{E}-16$ & $7.12 \mathrm{E}-12$ & 0.0004 & 4.19E-05 & $9.42 \mathrm{E}-05$ & 0.2305 & 0.61 & 0.017 & 0.5594 \\
\hline \multicolumn{10}{|c|}{ Correlation $2: \mathrm{b} 1 /\left(1+\exp \left(\mathrm{b} 2 *\right.\right.$ input $1+\mathrm{b} 3 *$ input $2+\mathrm{b} 4 *$ input $3+\mathrm{b} 5 *_{\text {input } 4+\mathrm{b} 6 * \text { input5 }+\mathrm{b} 7 * \text { input } 7+\mathrm{b} 8 * \text { input } 8))}$} \\
\hline & b1 & $\mathrm{b} 2$ & b3 & $\mathrm{b} 4$ & b5 & b6 & b7 & $\mathrm{b} 8$ & b9 \\
\hline Coefficient & 73.5949 & -0.0003 & 0.0724 & 0.1778 & 0.3241 & -1.6517 & 0.5688 & 1.4057 & - \\
\hline p-value & $1.67 \mathrm{E}-16$ & $1.68 \mathrm{E}-12$ & $2.13 \mathrm{E}-05$ & $9.60 \mathrm{E}-06$ & $2.69 \mathrm{E}-05$ & 0.0786 & 0.0022 & 0.0805 & - \\
\hline \multicolumn{10}{|c|}{ Correlation 3: b1/(1+ exp $(\mathrm{b} 2 *$ input $1+\mathrm{b} 3 *$ input $2+\mathrm{b} 4 *$ input $3+\mathrm{b} 5 *$ input $4+\mathrm{b} 6 *$ input5 $+\mathrm{b} 7 *$ input7 $))$} \\
\hline & b1 & $\mathrm{b} 2$ & b3 & $\mathrm{b} 4$ & b5 & b6 & b7 & $\mathrm{b} 8$ & b9 \\
\hline Coefficient & 73.3652 & -0.0003 & 0.0715 & 0.1612 & 0.3578 & -0.0147 & 0.6424 & - & - \\
\hline p-value & $9.54 \mathrm{E}-17$ & $1.68 \mathrm{E}-12$ & $4.33 \mathrm{E}-05$ & $2.88 \mathrm{E}-05$ & $6.65 \mathrm{E}-06$ & 0.8222 & 0.0009 & - & - \\
\hline \multicolumn{10}{|c|}{ Correlation $4: \mathrm{b} 1 /(1+\exp (\mathrm{b} 2 *$ input $1+\mathrm{b} 3 *$ input $2+\mathrm{b} 4 *$ input $3+\mathrm{b} 5 *$ input $4+\mathrm{b} 6 *$ input 7$))$} \\
\hline & b1 & $\mathrm{b} 2$ & b3 & b4 & b5 & b6 & b7 & b8 & b9 \\
\hline Coefficient & 73.3305 & -0.0003 & 0.0714 & 0.1612 & 0.3584 & 0.6414 & - & - & - \\
\hline p-value & $1.55 \mathrm{E}-17$ & $4.71 \mathrm{E}-13$ & $2.85 \mathrm{E}-05$ & $1.86 \mathrm{E}-05$ & $3.83 \mathrm{E}-06$ & 0.0007 & - & - & - \\
\hline \multicolumn{10}{|c|}{ Correlation $5: \mathrm{b} 1 /(1+\exp (\mathrm{b} 2 *$ input $1+\mathrm{b} 3 *$ input $2+\mathrm{b} 4 *$ input $3+\mathrm{b} 5 *$ input 4$))$} \\
\hline & $\mathrm{b} 1$ & $\mathrm{~b} 2$ & b3 & $\mathrm{b} 4$ & b5 & b6 & b7 & $\mathrm{b} 8$ & b9 \\
\hline Coefficient & 76.677 & -0.0002 & 0.0152 & 0.2564 & 0.2842 & - & - & - & - \\
\hline p-value & $1.24 \mathrm{E}-16$ & $6.82 \mathrm{E}-11$ & 0.0677 & $5.11 \mathrm{E}-36$ & $6.83 \mathrm{E}-08$ & - & - & - & - \\
\hline Coefficient & 75.8406 & -0.0002 & 0.251 & 0.3574 & - & - & - & - & - \\
\hline p-value & $8.06 \mathrm{E}-17$ & $1.02 \mathrm{E}-10$ & $3.11 \mathrm{E}-37$ & $4.11 \mathrm{E}-18$ & - & - & - & - & - \\
\hline
\end{tabular}


Table 6. Comparison of proposed correlations of 3 and 4 for prediction of outputs

\begin{tabular}{|c|c|c|c|c|c|c|c|c|c|c|c|c|}
\hline $\begin{array}{c}\mathrm{Ex} \\
\mathrm{p}\end{array}$ & $\begin{array}{c}\text { Input } \\
1 \\
\operatorname{Re} \\
\end{array}$ & $\begin{array}{c}\text { Input } 2 \\
\mathrm{Gr}_{\Delta \mathrm{T}} \times 10^{-} \\
6\end{array}$ & $\begin{array}{c}\text { Input } \\
3 \\
\operatorname{Pr} \\
\end{array}$ & $\begin{array}{c}\text { Input } \\
4 \\
\text { Bo } \\
\end{array}$ & $\begin{array}{c}\text { Input } \\
5 \\
\mathrm{f}\end{array}$ & $\begin{array}{c}\text { Input } \\
6 \\
\mathrm{f}_{\mathrm{o}} \\
\end{array}$ & $\begin{array}{c}\text { Input } \\
7 \\
\mu_{\mathrm{w}} / \mu_{\mathrm{b}} \\
\end{array}$ & $\begin{array}{c}\text { Input } \\
8 \\
\mathrm{f}_{\mathrm{vp}} \\
\end{array}$ & $\begin{array}{c}\text { Corr. } \\
3 \\
\mathrm{Nu}_{\mathrm{av}} \\
\end{array}$ & $\begin{array}{c}\text { Corr. } \\
4 \\
\mathrm{Nu}_{\mathrm{av}} \\
\end{array}$ & $\begin{array}{c}\text { Output } \\
1 \\
\mathrm{Nu}_{\mathrm{av}} \\
\end{array}$ & $\begin{array}{c}\text { Outpu } \\
\text { t } 2 \\
\mathrm{Nu}_{\mathrm{o}} \\
\end{array}$ \\
\hline $1 \mathrm{U}$ & 7513 & 4.730 & 4.970 & 0.138 & 0.029 & 0.033 & 0.510 & 0.028 & 26.300 & 25.401 & 24.300 & 49.390 \\
\hline $2 \mathrm{U}$ & 7391 & 2.110 & 5.060 & 0.114 & 0.031 & 0.033 & 0.700 & 0.031 & 43.158 & 43.469 & 41.600 & 49.057 \\
\hline $3 \mathrm{U}$ & 7200 & 0.988 & 5.220 & 0.061 & 0.032 & 0.033 & 0.840 & 0.032 & 49.613 & 50.166 & 46.800 & 48.397 \\
\hline $4 U$ & 7031 & 0.474 & 5.360 & 0.031 & 0.033 & 0.034 & 0.910 & 0.033 & 50.001 & 50.691 & 47.100 & 47,866 \\
\hline $5 \mathrm{U}$ & 6069 & 0.538 & 5.380 & 0.050 & 0.034 & 0.036 & 0.900 & 0.035 & 40.188 & 40.745 & 39.700 & 42.688 \\
\hline $6 \mathrm{U}$ & 5012 & 0.653 & 5.400 & 0.094 & 0.041 & 0.038 & 0.870 & 0.037 & 27.984 & 28.581 & 31.500 & 36.713 \\
\hline $7 \mathrm{U}$ & 4163 & 1.080 & 5.360 & 0.151 & - & - & - & - & - & - & \begin{tabular}{|l|}
16.600 \\
\end{tabular} & 31.499 \\
\hline $8 \mathrm{U}$ & 4143 & 0.405 & 5.380 & 0.089 & 0.041 & 0.040 & 0.920 & 0.039 & 26.418 & 26.601 & 24.800 & 31.000 \\
\hline $9 \mathrm{U}$ & 4990 & 0.304 & 5.430 & 0.047 & 0.039 & 0.038 & 0.940 & 0.038 & 32.470 & 33.019 & 33.800 & 36.541 \\
\hline $\begin{array}{l}10 \\
\mathrm{U}\end{array}$ & 5087 & 1.610 & 5.310 & 0.139 & 0.033 & 0.038 & 0.750 & 0.035 & 21.951 & 21.001 & 20.100 & 36.949 \\
\hline $\begin{array}{l}11 \\
\mathrm{U}\end{array}$ & 4210 & 2.060 & 5.290 & 0.249 & - & - & - & - & - & - & 14.300 & 31.567 \\
\hline $\begin{array}{l}12 \\
\mathrm{U}\end{array}$ & 6218 & 1.050 & 5.240 & 0.088 & 0.035 & 0.035 & 0.820 & 0.034 & 38.603 & 39.210 & 37.600 & 43.169 \\
\hline $\begin{array}{l}13 \\
\mathrm{U}\end{array}$ & 5268 & 3.130 & .100 & 0.227 & - & - & - & - & - & - & 17.800 & 37.553 \\
\hline $\begin{array}{l}14 \\
\mathrm{U}\end{array}$ & 4391 & 3.240 & 5.040 & 0.497 & - & - & - & - & - & - & 19.300 & 35.874 \\
\hline $\begin{array}{l}15 \\
U\end{array}$ & 4578 & 4.620 & 4.820 & 0.771 & - & - & - & - & - & - & 28.100 & 33.733 \\
\hline $\begin{array}{l}16 \\
\mathrm{U}\end{array}$ & 5462 & 4.480 & 4.900 & 0.398 & 0.039 & 0.037 & 0.470 & 0.030 & 20.215 & 20.202 & 22.700 & 38.151 \\
\hline $\begin{array}{l}17 \\
\mathrm{U}\end{array}$ & 6341 & 2.820 & 5.120 & 0.140 & 0.031 & 0.035 & 0.650 & 0.032 & 27.102 & 26.458 & 24.000 & 43.557 \\
\hline $\begin{array}{l}18 \\
\mathrm{U}\end{array}$ & 6689 & 5.540 & 4.810 & 0.270 & 0.044 & 0.034 & 0.460 & 0.028 & 23.891 & 24.866 & 24.800 & 44.604 \\
\hline $\begin{array}{l}19 \\
\mathrm{U}\end{array}$ & 4658 & 2,590 & 190 & 0,259 & - & - & - & - & - & - & 16.300 & 34.100 \\
\hline $\begin{array}{l}20 \\
\mathrm{U}\end{array}$ & 4837 & 3,540 & 4,970 & 0,441 & 0,047 & 0,038 & 0,540 & 0,033 & 21.746 & 22.816 & 21.000 & 34.711 \\
\hline $\begin{array}{l}21 \\
\mathrm{U}\end{array}$ & 5220 & 6,930 & 4,550 & 0,920 & - & - & - & - & - & - & 27.800 & 36.010 \\
\hline $\begin{array}{l}22 \\
D\end{array}$ & 5154 & 1,090 & 5,230 & 0,181 & 0,033 & 0,040 & 0,820 & 0,039 & 35.438 & 34.989 & 40.200 & 37.051 \\
\hline $\begin{array}{l}23 \\
D\end{array}$ & 07 & 720 & 5,060 & 0,279 & 0,031 & 0,040 & 0,760 & 0,038 & 41.410 & 41.112 & 42.000 & 37.702 \\
\hline $\begin{array}{l}24 \\
D\end{array}$ & 5646 & 3,140 & 4,740 & 0,498 & - & - & - & - & - & - & 48.000 & 38.710 \\
\hline $\begin{array}{l}25 \\
D\end{array}$ & 5956 & 4,950 & 4,430 & 0,730 & - & - & - & - & - & - & 52.100 & 39.710 \\
\hline $\begin{array}{l}26 \\
D\end{array}$ & 5112 & 0,740 & 5,280 & 0,119 & 0,340 & 0,402 & 0,870 & 0,394 & 38.073 & 38.103 & 38.000 & 37.037 \\
\hline $\begin{array}{l}27 \\
D\end{array}$ & 4653 & 0,815 & 5,190 & 0,174 & 0,033 & 0,041 & 0,870 & 0,040 & 37.583 & 37.143 & 36.000 & 34.059 \\
\hline $\begin{array}{l}28 \\
D\end{array}$ & 4366 & 0,812 & 5,290 & 0,204 & - & - & - & - & 0.259 & 0.150 & 34.500 & 32.609 \\
\hline $\begin{array}{l}29 \\
D\end{array}$ & 4339 & 0,433 & 5,330 & 0,106 & 0,037 & 0,042 & 0,920 & 0,041 & 31.486 & 31.170 & 32300 & 32.528 \\
\hline
\end{tabular}


Table 6. (Cont.). Comparison of proposed correlations of 3 and 4 for prediction of outputs

\begin{tabular}{|c|c|c|c|c|c|c|c|c|c|c|c|c|}
\hline $\begin{array}{c}\text { Ex } \\
p\end{array}$ & $\begin{array}{c}\text { Input } \\
1 \\
\mathrm{Re} \\
\end{array}$ & $\begin{array}{c}\text { Input } 2 \\
\mathrm{Gr}_{\Delta \mathrm{T}} \times 10^{-} \\
6\end{array}$ & $\begin{array}{c}\text { Input } \\
3 \\
\operatorname{Pr} \\
\end{array}$ & $\begin{array}{c}\text { Input } \\
4 \\
\text { Bo }\end{array}$ & $\begin{array}{c}\text { Input } \\
5 \\
\mathrm{f}\end{array}$ & $\begin{array}{c}\text { Input } \\
6 \\
\mathrm{f}_{\mathrm{o}} \\
\end{array}$ & $\begin{array}{c}\text { Input } \\
7 \\
\mu_{\mathrm{w}} / \mu_{\mathrm{b}} \\
\end{array}$ & $\begin{array}{c}\text { Input } \\
8 \\
\mathrm{f}_{\mathrm{vp}} \\
\end{array}$ & $\begin{array}{c}\text { Corr. } \\
3 \\
\mathrm{Nu}_{\mathrm{av}} \\
\end{array}$ & $\begin{array}{c}\text { Corr. } \\
4 \\
\mathrm{Nu}_{\mathrm{av}} \\
\end{array}$ & $\begin{array}{c}\text { Output } \\
1 \\
\mathrm{Nu}_{\mathrm{av}} \\
\end{array}$ & $\begin{array}{c}\text { Outpu } \\
\text { t } 2 \\
\mathrm{Nu}_{\mathrm{o}} \\
\end{array}$ \\
\hline 30D & 4671 & 0.424 & 5.310 & 0.083 & 0.037 & 0.041 & 0.920 & 0.041 & 33.750 & 33.620 & 34.200 & 34.476 \\
\hline $31 \mathrm{D}$ & 4873 & 1.260 & 5.160 & 0.260 & 0.032 & 0.041 & 0.810 & 0.039 & 38.860 & 38.507 & 38.100 & 34.890 \\
\hline $32 \mathrm{D}$ & 4527 & 1.350 & 5.080 & 0.334 & 0.032 & 0.042 & 0.800 & 0.040 & 41.569 & 41.342 & 37.400 & 33.156 \\
\hline $33 \mathrm{D}$ & 4999 & 1.960 & 4.910 & 0.390 & - & - & - & - & 1.173 & 0.802 & 41.400 & 35.506 \\
\hline $34 \mathrm{D}$ & 333 & .530 & .130 & 0.150 & .030 & 0.038 & 0.780 & 0.036 & 43.376 & 43.082 & 47.100 & 43.530 \\
\hline $35 \mathrm{D}$ & 7357 & 1.370 & 5.180 & 0.088 & 0.030 & 0.036 & 0.790 & 0.035 & 48.745 & 48.687 & 52.500 & 49.203 \\
\hline $36 \mathrm{D}$ & 8468 & 1.250 & 5.150 & 0.055 & 0.029 & 0.034 & 0.810 & 0.033 & 59.139 & 58.894 & 58,300 & 55.000 \\
\hline 37D & 8640 & 1.900 & 5.030 & 0.082 & 0.028 & 0.034 & 0.750 & 0.032 & 59.175 & 58.796 & 59,800 & 55.422 \\
\hline 38D & 7582 & 2.140 & 5.000 & 0.135 & 0.028 & 0.036 & 0.730 & 0.034 & 52.313 & 52.001 & 54.800 & 49.864 \\
\hline 39D & 6606 & 2.470 & 4.890 & 0.234 & - & - & - & - & - & - & 50.700 & 44.396 \\
\hline $40 \mathrm{D}$ & 5048 & 3.010 & 4.730 & 0.637 & - & - & - & - & - & - & 44.200 & 35.474 \\
\hline $41 \mathrm{D}$ & 4868 & 3.890 & 4.670 & 0.756 & - & - & - & - & - & - & 43.800 & 34.272 \\
\hline $42 \mathrm{D}$ & 7702 & 2.780 & 4.910 & 0.173 & - & - & - & - & - & - & 56.600 & 50.311 \\
\hline $43 \mathrm{D}$ & 6710 & 3.190 & 4.800 & 0.302 & - & - & - & - & - & - & 52.800 & 44.708 \\
\hline 44D & 5293 & 4.970 & 4.600 & 0.782 & - & - & - & - & - & - & 47.200 & 36.141 \\
\hline $45 \mathrm{D}$ & 5020 & 4.140 & 4.510 & 0.983 & - & - & - & - & - & - & 46.900 & 34.766 \\
\hline $46 \mathrm{D}$ & 8740 & 2.500 & 4.970 & 0.108 & - & - & - & - & - & - & 61.400 & 55.818 \\
\hline $47 \mathrm{D}$ & 6993 & 4.340 & 4.570 & 0.390 & - & - & - & - & - & - & 55.800 & 45.551 \\
\hline 48D & 7930 & 3.730 & 4.750 & 0.223 & - & - & - & - & - & - & 58.700 & 50.911 \\
\hline 49D & 9059 & 3.410 & 4.770 & 0.139 & - & - & - & - & - & - & 63.700 & 56.723 \\
\hline $50 \mathrm{D}$ & 5518 & 5.150 & 4.380 & 0.987 & - & - & - & - & - & - & 50.800 & 37.216 \\
\hline 51D & 5251 & 5.600 & 4.280 & 1.273 & - & - & - & - & - & - & 50.400 & 35.493 \\
\hline
\end{tabular}

It should be noted that this paper should be evaluated as an extension of authors' previous study [18]. Detailed information on ANN analyses can be obtained from that study [18].

\section{CONCLUSION}

This investigation studied some single-phase flow characteristics of water during transition and turbulent flow in a vertical tube. Parlatan et al.'s [4] experimental data were used in the analyses. The purpose of this numerical study was to illustrate ANN method's capability to determine Nusselt numbers, effect of dimensionless numbers as inputs on Nusselt numbers as outputs, and propose various empirical correlations using the inputs. The validation process of the results revealed the importance of dimensionless numbers on the measured pipe length averaged Nusselt numbers and forced convection's Nusselt numbers according to aiding and opposing flow types. There are few research studies on such cases in the literature. For this reason, the results of this study are expected to fill a gap in the literature. Twelve different correlations were proposed using inputs of the numerical model for the outputs separately. The best correlations' figures were plotted within the deviation of $\pm 5 \%$. Some dimensionless numbers regarding buoyancy influence $\left(\mathrm{Gr}_{\square \mathrm{T}}\right)$ and the effects of temperature-dependent viscosity variations $\left(\mu_{\mathrm{w}} / \mu_{\mathrm{b}}\right)$ were added into both ANN analyses.

\section{ACKNOWLEDGEMENT}

The second author would like to thank King Mongkut's University of Technology Thonburi for the support during his research in Thailand. The fifth author would like to express his appreciation to the National Science and Technology for Development (NSTDA 2013) and the National Research University (NRU 2014) for the support.

\section{NOMENCLATURE}

ANN Artificial Neural Network 


$\begin{array}{ll}\mathrm{Bo} & \text { Buoyancy number } \\ \mathrm{D} & \text { Inside diameter of tube, } \mathrm{m} \\ \mathrm{f} & \text { Darcy friction factor } \\ \mathrm{f}_{\mathrm{o}} & \text { Isothermal friction factor in forced convection } \\ \mathrm{g} & \text { Gravitational acceleration, } \mathrm{m} \mathrm{s}^{-2} \\ \mathrm{Gr} & \text { Grashof number } \\ \mathrm{L} & \text { Test section length } \\ \mathrm{Nu} & \text { Nusselt number } \\ \mathrm{Nu} & \text { Tube length averaged Nusselt number } \\ \mathrm{Nu} & \text { Nusselt number in forced convection } \\ \mathrm{Pr} & \text { Prandtl number } \\ \mathrm{Re} & \text { Reynolds number } \\ \mathrm{q} " & \text { Net wall heat flux, } \mathrm{W} \mathrm{m}^{-2} \\ \mathrm{~T}_{\mathrm{b}} & \text { Bulk temperature, }{ }^{\circ} \mathrm{C} \\ \mathrm{T}_{\mathrm{w}} & \text { Wall temperature, }{ }^{\circ} \mathrm{C} \\ \Delta \mathrm{P} & \text { Pressure drop, Pa } \\ \beta & \text { Thermal expansion coefficient, } 1 \mathrm{~K}^{-1} \\ v & \text { Kinematic viscosity, } \mathrm{m}^{2} \mathrm{~s}^{-1} \\ \alpha & \text { Thermal diffusivity } \\ \rho & \text { Density, kg } \mathrm{m}^{-3}\end{array}$

\section{REFERENCES}

[1] Hiroaki, T., Ayao, T., Masaru, H., Nuchi, N. (1973). Effects of buoyancy and of acceleration owing to thermal expansion on forced turbulent convection in vertical circular tubes - criteria of the effects, velocity and temperature profiles, and reverse transition from turbulent to laminar flow. International Journal of Heat and Mass Transfer, 16(6), 1267-1288.

[2] Easby, J. P. (1978). The effect of buoyancy on flow and heat transfer for a gas passing down a vertical pipe at low turbulent Reynolds numbers. International Journal of Heat and Mass Transfer, 21(6), 791-801.

[3] Saylor, P. E., \& Joye, D. D. (1991). Hydrostatic correction and pressure drop measurement in mixed convection heat transfer in a vertical tube. Industrial \& Engineering Chemistry Research, 30(4), 784-788.

[4] Parlatan, Y., Todreas, N. E., Driscoll, M. J. (1996). Buoyancy and property variation effects in turbulent mixed convection of water in vertical tubes. Journal of heat transfer, 118(2), 381-387.

[5] You, J., Yoo, J. Y., Choi, H. (2003). Direct numerical simulation of heated vertical air flows in fully developed turbulent mixed convection. International Journal of Heat and Mass Transfer, 46(9), 1613-1627.

[6] Joye, D. D. (2003). Pressure drop correlation for laminar, mixed convection, aiding flow heat transfer in a vertical tube. International journal of heat and fluid flow, 24(2), 260-266.

[7] Busedra, A. A., Soliman, H. M. (1999). Analysis of laminar mixed convection in inclined semicircular ducts under buoyancy-assisted and-opposed conditions. Numerical Heat Transfer: Part A: Applications, 36(5), $527-544$. [8] Voicu, I., Maré, T., Galanis, N., Miriel, J., Colda, I. (2007). Mixed convection in a vertical double pipe heat exchanger. International Journal of Thermal Sciences, 46(6), 540-550.

[9] Kang, G. U., \& Chung, B. J. (2012). Influence of the height-to-diameter ratio on turbulent mixed convection in vertical cylinders. Heat and Mass Transfer, 48(7), 1183-1191.

[10] Tam, L. M., Ghajar, A. J., \& Tam, H. K. (2008). Contribution analysis of dimensionless variables for laminar and turbulent flow convection heat transfer in a horizontal tube using artificial neural network. Heat Transfer Engineering, 29(9), 793-804.

[11] Selimefendigil, F., \& Öztop, H. F. (2014). Estimation of the mixed convection heat transfer of a rotating cylinder in a vented cavity subjected to nanofluid by using generalized neural networks. Numerical Heat Transfer, Part A: Applications, 65(2), 165-185.

[12] Balcilar, M., Dalkilic, A. S., \& Wongwises, S. (2011). Artificial neural network techniques for the determination of condensation heat transfer characteristics during downward annular flow of R134a inside a vertical smooth tube. International Communications in Heat and Mass Transfer, 38(1), 75-84. 
[13] Balcilar, M., Dalkilic, A. S., Suriyawong, A., Yiamsawas, T., \& Wongwises, S. (2012). Investigation of pool boiling of nanofluids using artificial neural networks and correlation development techniques. International Communications in Heat and Mass Transfer, 39(3), 424-431.

[14] Balcilar, M., Dalkilic, A. S., Agra, O., Atayilmaz, S. O., Wongwises, S. (2012). A correlation development for predicting the pressure drop of various refrigerants during condensation and evaporation in horizontal smooth and micro-fin tubes. International Communications in Heat and Mass Transfer, 39(7), 937-944.

[15] Kayaci, N., Balcilar, M., Tabatabaei, M., Celen, A., Yildiz, O., Dalkilic, A. S., Wongwises, S. (2013). Determination of the Single-Phase Forced Convection Heat Transfer Characteristics of TiO2Nanofluids Flowing in Smooth and Micro-Fin Tubes by Means of CFD and ANN Analyses. Current Nanoscience, 9(1), 61-80.

[16] Balcilar, M., Aroonrat, K., Dalkilic, A. S., Wongwises, S. (2013). A numerical correlation development study for the determination of Nusselt numbers during boiling and condensation of R134a inside smooth and corrugated tubes. International Communications in Heat and Mass Transfer, 48, 141-148.

[17] Balcilar, M., Aroonrat, K., Dalkilic, A. S., Wongwises, S. (2013). A generalized numerical correlation study for the determination of pressure drop during condensation and boiling of R134a inside smooth and corrugated tubes. International Communications in Heat and Mass Transfer, 49, 78-85.

[18] Balcilar, M., Dalkilic, A. S., Aroonrat, K.,Wongwises, S. (2014). Neural network based analyses for the determination of evaporation heat transfer characteristics during downward flow of R134a inside a vertical smooth and corrugated tube. Arabian Journal for Science and Engineering, 39(2), 1271-1290.

[19] Balcilar, M., Dalkilic, A. S., Sonmez, A. C., Wongwises, S. (2014). Classification of in-tube boiling R134a data belonging to the smooth and corrugated tubes. International Communications in Heat and Mass Transfer, 53, 185-194. 